\title{
Comparison of Vibrational Relaxation Modeling for Strongly Non-Equilibrium Flows
}

\author{
Timothy R. Deschenes, Matthew Braunstein ${ }^{\dagger}$ \\ Spectral Sciences, Inc., Burlington, MA, 01824 \\ and \\ Iain D. Boyd ${ }^{\ddagger}$ \\ Department of Aerospace Engineering, University of Michigan, Ann Arbor, MI, 48109
}

\begin{abstract}
The detailed description of the vibrational energy content is important for many gas flow processes including experimental gas measurement techniques, shock layer vibrationdissociation coupling, and vibrational energy freezing in strong expansions. Various vibrational relaxation models have been developed for use within the direct simulation Monte Carlo (DSMC) method. This study compares the Larsen-Borgnakke and Forced Harmonic Oscillator vibrational relaxation models with detailed quasi-classical trajectory (QCT) results for an atom-diatom system with focus on the effects of the form of the relaxation model. These QCT results have been extensively compared with measured data and provide a unique opportunity to evaluate these inelastic models. This work also compares the results from each model for a relaxation problem with vibrational excitation. Even when the two models have consistent vibrational collision numbers to match bulk temperature predictions for relaxation problems, the predicted evolution of the vibrational distribution function differs. This disagreement results in different prediction of radiative emission spectra.
\end{abstract}

\section{Introduction}

Excitation and quenching of vibrational energy modes through collision relaxation is an important process in a wide range of high speed flows. High temperature shock layers that form in front of hypersonic vehicles can lead to significant vibrational excitation. Due to the relatively slow vibrational excitation rates, the vibrational energy may only be partially excited compared to the translational and rotational energy modes within most of the shock layer and the vibrational energy distribution function (VEDF) may not be characterized by an average mode temperature due to the strong non-equilibrium effects. In high expansion flows, the vibrational energy may remain frozen at near-stagnation conditions and large regions of nozzle flows may experience highly non-equilibrium VEDFs. This non-equilibrium VEDF has a strong effect on the coupled vibration-dissociation of molecular species. In addition, the non-equilibrium VEDF has a strong effect on measured radiative emission that is used to estimate vibrational energy content in experimental systems. The non-equilibrium VEDF is also important to evaluate fundamental gas dynamic properties, such as the ratio of specific heats and thermal conductivity.

Many vibrational relaxation models have been developed to use in both continuum flows for use in traditional Computational Fluid Dynamics (CFD) and non-continuum flows for use with rarefied flow descriptions, such as the direct simulation Monte Carlo (DSMC) method. At non-continuum (or rarefied) conditions, the non-equilibrium effects of vibrational relaxation become even more important as there are insufficient collisions to equilibrate the fast relaxation of the velocity distribution function (VDF) as well as the relatively slow relaxation of the VEDF. Recently Boyd and Josyula, ${ }^{1}$ have compared standard DSMC vibrational

*Senior Scientist, Member AIAA. Email: tdeschenes@spectral.com.

†Senior Technical Fellow.

${ }^{\ddagger}$ James E. Knott Professor, Fellow AIAA. 
relaxation approaches ${ }^{2,3}$ with a high-fidelity vibrational relaxation model. ${ }^{4}$ They also have compared the high-fidelity results with thermally-averaged results obtained by Billing and Fisher. ${ }^{5}$

In this work, we extend the previous work ${ }^{1}$ by comparing both low- and high-fidelity results to stateresolved quasi-classical trajectory (QCT) results from Braunstein and Duff ${ }^{6}$. These results have been extensively compared with experimental measurements ${ }^{7}$ and provide a unique opportunity to evaluate a wide range of vibrational inelastic collision models. First, we present the ro-vibrationally resolved cross sections of Braunstein and Duff. Then, we describe the low- and high-fidelity models that are used within the DSMC method with focus on the inherent form that dictates the VEDF that is sampled in vibrationally inelastic collisions. Next, we compare the models to available data from the QCT modeling for vibrational-translational energy exchange for an atom-diatom system. In particular, we assess the models by comparing the predicted VEDF during a vibrational inelastic collision at a given initial state and collision velocity. Finally, the two models are applied in the DSMC method to predict vibrational excitation in an adiabatic relaxation case, where both models are tuned to agree in the bulk relaxation process. This isolates the effect of the model form on the predicted evolution of the VEDF and associated radiative emission spectra. Detailed comparison of the evolution of the VEDF and emission spectra are compared for each vibrational relaxation model. The paper closes with a summary and conclusions.

\section{Available State-to-State Transition Data}

In this section, we present model results from quasi-classical trajectory (QCT) calculations of Braunstein and Duff. ${ }^{6}$ These results have been extensively compared with experimental measurements ${ }^{7}$ and provide an opportunity to evaluate a wide range of vibrational relaxation models. We present the ro-vibrational cross sections for a number of relative collision velocities and compare the post vibrationally-inelastic VEDF (PVI-VEDF), which is described in Section III, at selected collision classes.

In Braunstein and Duff, ${ }^{6}$ the potential energy surfaces of the three lowest electronic triplet states of $\mathrm{CO}_{2}$ which lead to $\mathrm{O}\left({ }^{3} \mathrm{P}\right)+\mathrm{CO}\left({ }^{1} \Sigma^{+}\right),{ }^{3} A^{\prime}, 1^{3} A^{\prime \prime}$, and $2^{3} A^{\prime \prime}$, were computed at the complete- active-spaceself-consistent-field plus second-order perturbation theory (CASSCF-MP2) level with a modest $631+\mathrm{G}(d)$ basis. Potential energy surfaces were fit with a global functional form. These potential energy surfaces were used to run classical trajectory calculations for ro-vibrationally resolved $\mathrm{O}\left({ }^{3} \mathrm{P}\right)+\mathrm{CO}\left({ }^{1} \Sigma^{+}\right)$collisions. When $\mathrm{O}+\mathrm{CO}$ collide, there is direct collisional excitation of the $\mathrm{CO}$ and there is also reaction where the incoming $\mathrm{O}$ exchanges with the $\mathrm{O}$ in the reactant $\mathrm{CO}$. Both collisional excitation and reaction channels lead to vibrational excitation of the product $\mathrm{CO}$. The QCT results presented below include both reactive and nonreactive channels summed together. Their results agree well with available angle and energy resolved cross section measurements ${ }^{7}$ except at low temperature. Favorable comparisons have been made with measured vibrational excitation cross sections and infrared emission spectra of the nascent $\mathrm{CO}$ products at $3.4 \mathrm{eV}$ collision energy. ${ }^{7}$

It is important to note that the methods used to generate these results are unable to accurately predict the vibrationally elastic collision cross-section. In this study, a separate total cross section model is used in conjunction with the QCT results to form an overall relaxation rate. For this work, the vibrationally elastic cross section is derived from the hard sphere model and has no effect on the study of the PVI-VEDF. These results provide an opportunity to evaluate a wide range of inelastic vibration models.

Figure 1 shows the post vibrationally-inelastic ro-vibrational energy distribution function (RVEDF) while varying the initial vibrational level and sampling the rotational energy from a Boltzmann distribution specified by a temperature of the $\mathrm{CO}$ molecule at two relative velocities. Figures 1(a), (c), and (e) show the post vibrationally-inelastic RVEDF at moderate thermal collision velocities of $4 \mathrm{~km} / \mathrm{s}$ (most probable collision velocity for CO-O at $T_{\mathrm{TRA}} \approx 13,000 \mathrm{~K}$ ), while Figs. $1(\mathrm{~b}),(\mathrm{d})$, and (f) show the RVEDF at a hyperthermal collision velocity of $10 \mathrm{~km} / \mathrm{s}$. Since the current work focuses on assessing vibrational relaxation models, we sum over the final rotational states. For reference, the energy spacing between the first excited and ground vibrational state is equivalent to the translational collision energy between the $\mathrm{CO}+\mathrm{O}$ system with a relative velocity of about $2.2 \mathrm{~km} / \mathrm{s}$.

Figure 2 shows the PVI-VEDF at a relative collision velocity of $4 \mathrm{~km} / \mathrm{s}$ that was calculated by summing over all possible rotational states in Figs. 1(a), (c), and (e). For the pure excitation case $\left(v_{i}=0\right)$, the available total energy is small enough to restrict the VEDF to the first two excited states. For the combined excitation/quenching cases $\left(v_{i}=4\right)$, there is a greater probability of a quenching process than a vibrationally excited collision. This is expected because the initial vibrational energy exceeds $60 \%$ of the total collisional 
energy. This VEDF is nearly linear in vibrational quanta. Additionally we see that the PVI-VEDF remains nearly the same as the initial rotational temperature is changed. This gives confidence that vibrational relaxation models may not require other internal energy states as inputs to maintain physical accuracy. This is the case for both vibrational relaxation models studied in this work.

Figure 3 show the PVI-VEDF at a relative collision velocity of $10 \mathrm{~km} / \mathrm{s}$ that was calculated by integrating over all possible rotational states in Figs. 1(b), (d), and (f). At this collision velocity, we see the pure excitation case $\left(v_{i}=0\right)$ fills many of the excited levels with measurable cross-sections up to $v_{f}=17$. Consistent with the lower relative collision velocity case, the mixed excitation/quenching results $\left(v_{i}=4\right)$ remain similar at the two initial rotational temperatures. However, the PVI-VEDF is split nearly equally between excitation and quenching with the jump of \pm 1 quanta being the most probable final state.

\section{Vibrational Relaxation Models}

Modeling of collisional vibrational relaxation within the DSMC method can be described by two steps within the collision process. First, a decision of whether vibrational relaxation should occur is determined using the vibrational cross section, $\sigma_{v i b}$, which may be a function of many collision pair parameters such as initial vibrational energy content, initial rotation energy content, and relative collision energy. This vibrational cross section is then compared to the total cross section, $\sigma_{t o t}$, to form a probability of vibrational energy exchange that is used within a Monte-Carlo algorithm. For a given collision, if a vibrational energy transfer process is selected, then post-exchange vibrational energy states are selected from a VEDF, which, in this work, is termed the post vibrationally-inelastic VEDF (PVI-VEDF), which again can be dependent on a wide range of parameters such as initial vibrational energy content of each collision pair, initial rotational energy content of each collision pair, and relative collision energy. Low-fidelity models typically require very little information to predict the vibrationally inelastic cross section and post inelastic vibrational populations, while high-fidelity models differentiate many collision classes through state-resolved vibrational cross sections and PVI-VEDF. In this description, all units are in SI unless otherwise noted.

\section{A. Low-Fidelity Vibrational Relaxation Models}

\section{Collision Number Selection}

Typically, low-fidelity vibrational relaxation models form the probability of vibrationally inelastic cross section, $\phi_{t o t}$, which is the ratio of vibrational cross section to total cross section. A constant value based on the average relaxation time can be applied to each collision. Alternatively, the probability can be based on a mix of collisional values or macroscopic information to match measured relaxation rates. This parameter can be freely chosen to match bulk relaxation and has little effect on the overall model form. References 8 and 9 provide further details of available collision number models.

\section{Post Vibrationally Inelastic Population Sampling}

After a vibrationally inelastic collision is selected, energy is distributed among available translational and vibrational energy modes using a modified version of the Larsen-Borgnakke ${ }^{2,10}$ (LB) procedure that accounts for quantized vibrational levels. For example, the LB PVI-VEDF for a harmonic oscillator, $f\left(\varepsilon_{\mathrm{VIB}}\right)$, can be defined by Eq. 1 where $\varepsilon_{\mathrm{VIB}}$ is the vibrational energy, $E_{c}$ is the total collision energy, $i$ is the vibrational quantum number, $k$ is the Boltzmann constant, $\theta_{\mathrm{VIB}}$ is the characteristic temperature for vibration, $\eta_{\mathrm{TRA}}$ is the number of translational degrees of freedom, and $\delta$ is the Dirac delta function. This model includes the effect of the initial vibrational energy in aggregate, but cannot differentiate between a high velocity impact collision with a low initial vibrational energy from a low velocity collision with a high initial vibrational energy level.

$$
f_{\mathrm{LB}}\left(\varepsilon_{\mathrm{VIB}}\right) \sim\left(1-\frac{\varepsilon_{\mathrm{VIB}}}{E_{c}}\right)^{\eta_{T R A}-1} \delta\left(\varepsilon_{\mathrm{VIB}}-i k \theta_{\mathrm{VIB}}\right)
$$

\section{B. High-Fidelity Vibrational Relaxation Model}

In this work, the high-fidelity vibrational relaxation model uses the vibrationally specific transition probabilities of the Forced Harmonic Oscillator (FHO) model described by Adamovich et al. ${ }^{4}$ For example, the 


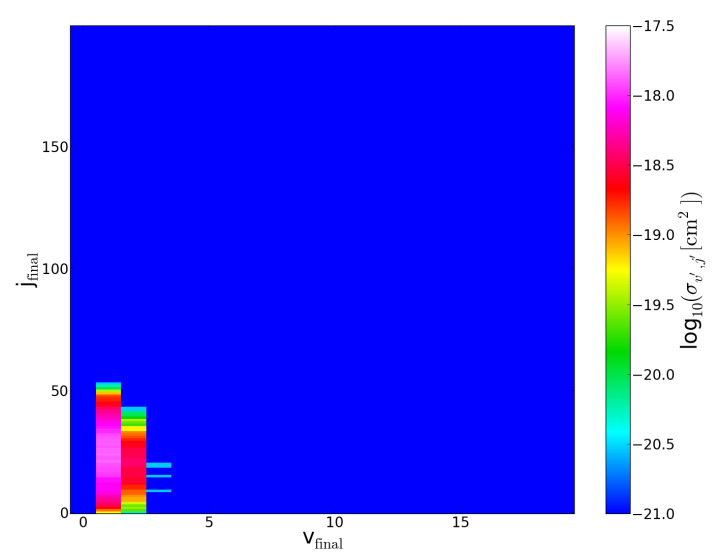

(a) $g_{\mathrm{rel}}=4 \mathrm{~km} \mathrm{~s}^{-1}, v_{\mathrm{i}}=0, T_{\mathrm{ROT}, \mathrm{i}}=300 \mathrm{~K}$

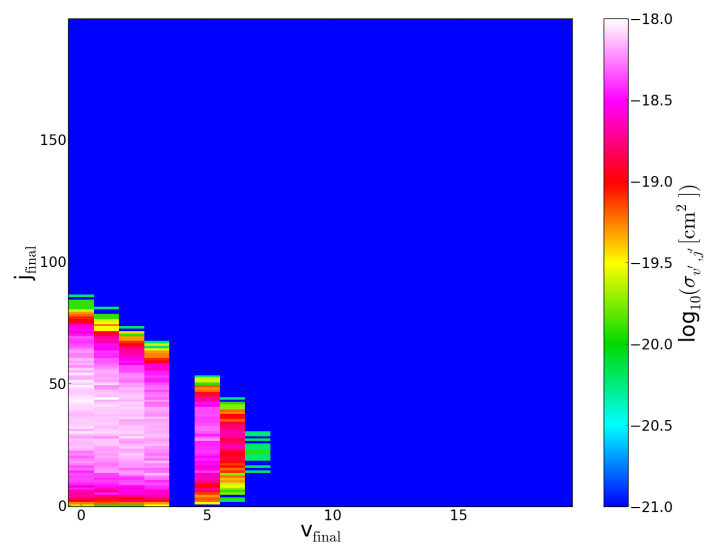

(c) $g_{\mathrm{rel}}=4 \mathrm{~km} \mathrm{~s}^{-1}, v_{\mathrm{i}}=4, T_{\mathrm{ROT}, \mathrm{i}}=300 \mathrm{~K}$

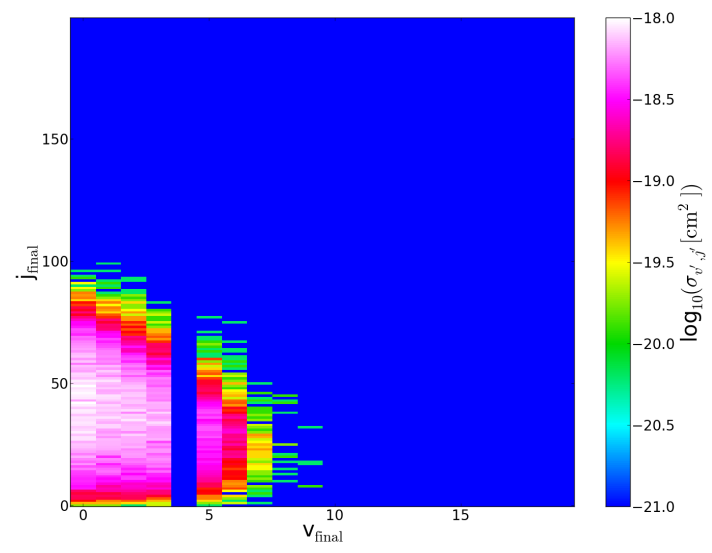

(e) $g_{\mathrm{rel}}=4 \mathrm{~km} \mathrm{~s}^{-1}, v_{\mathrm{i}}=4, T_{\mathrm{ROT}, \mathrm{i}}=2,000 \mathrm{~K}$

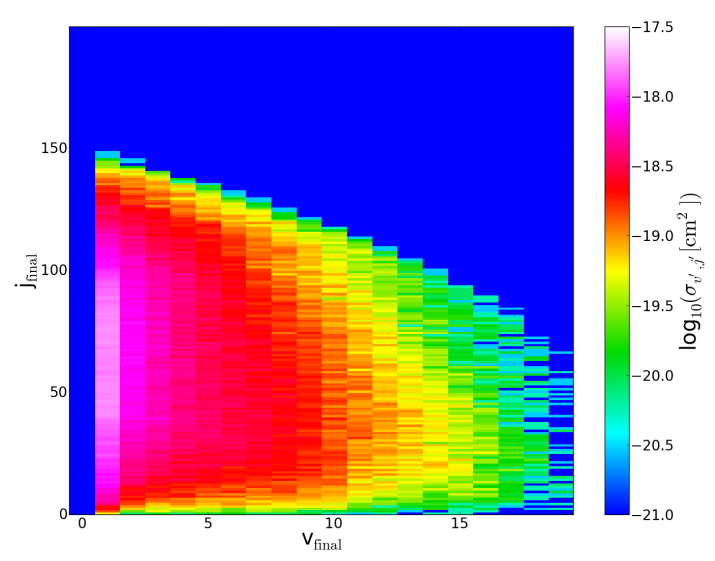

(b) $g_{\mathrm{rel}}=10 \mathrm{~km} \mathrm{~s}^{-1}, v_{\mathrm{i}}=0, T_{\mathrm{ROT}, \mathrm{i}}=300 \mathrm{~K}$

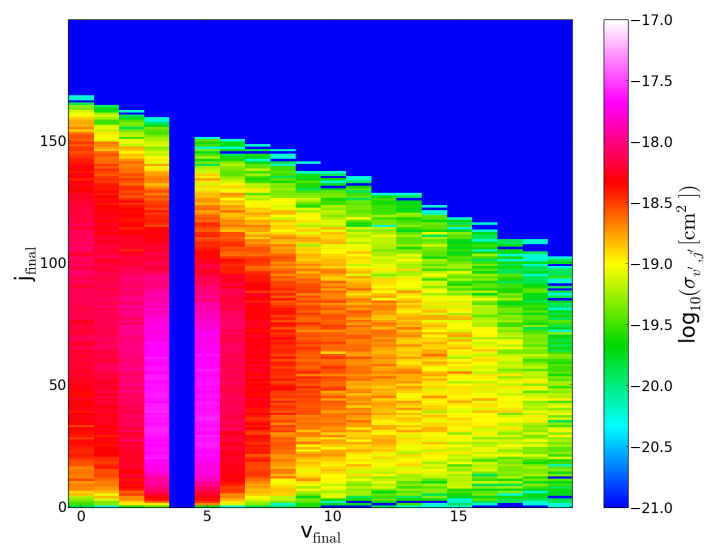

(d) $g_{\mathrm{rel}}=10 \mathrm{~km} \mathrm{~s}^{-1}, v_{\mathrm{i}}=4, T_{\mathrm{ROT}, \mathrm{i}}=300 \mathrm{~K}$

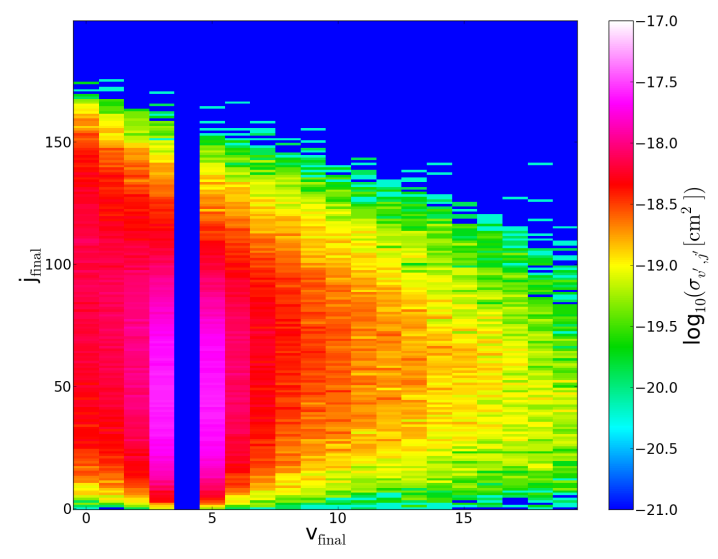

(f) $g_{\mathrm{rel}}=10 \mathrm{~km} \mathrm{~s}^{-1}, v_{\mathrm{i}}=4, T_{\mathrm{ROT}, \mathrm{i}}=2,000 \mathrm{~K}$

Figure 1: Predicted ro-vibrational distribution function from QCT results for post inelastic collision states given the relative velocity and initial vibrational level and rotational temperature of the $\mathrm{CO}$ molecule.

\section{4 of 15}

American Institute of Aeronautics and Astronautics

Distribution A: Approved for public release; distribution unlimited. 


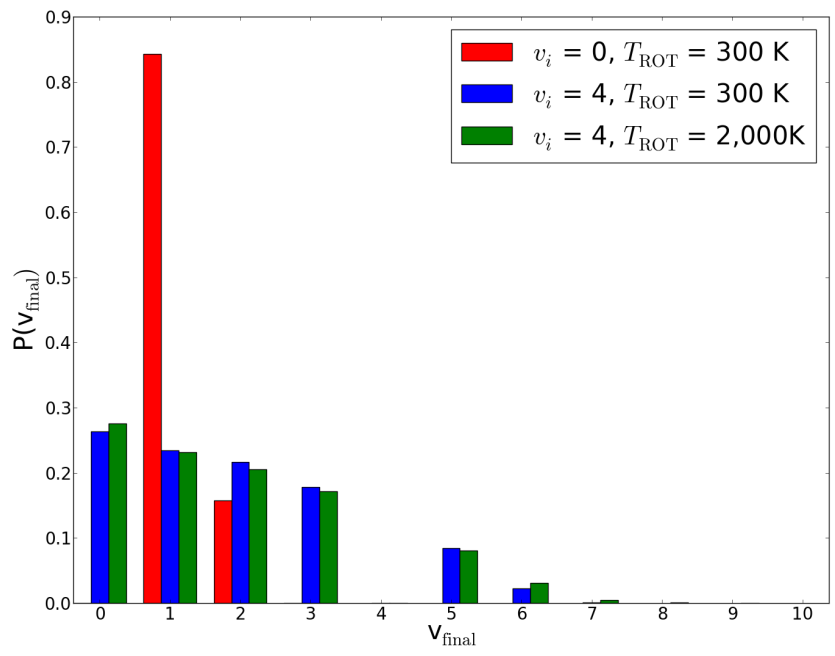

Figure 2: Predicted post-inelastic vibrational distribution function from QCT results with an initial relative velocity of $4 \mathrm{~km} / \mathrm{s}$ and specified initial vibrational quantum number and rotational temperature for the $\mathrm{CO}$ molecule. Results are normalized such that the integral of the VEDF is unity.

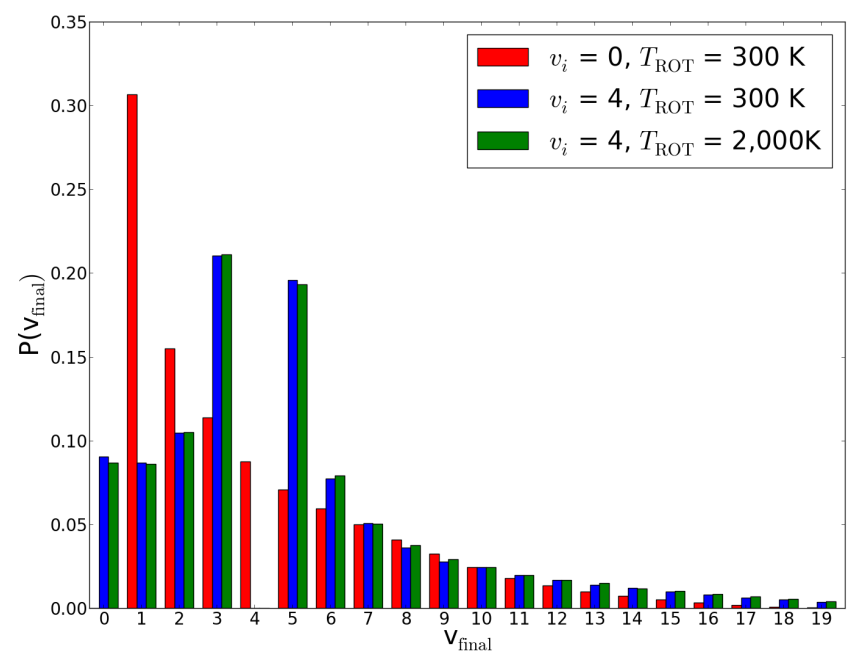

Figure 3: Predicted vibrational distribution function from QCT results with an initial relative velocity of $10 \mathrm{~km} / \mathrm{s}$ and specified initial vibrational quantum number and rotational temperature for the CO molecule. Results are normalized such that the integral of the VEDF is unity.

\section{5 of 15}

American Institute of Aeronautics and Astronautics

Distribution A: Approved for public release; distribution unlimited. 
probability of vibrational relaxation for an atom-diatom collision is described by Eq. 2 where $i$ is the initial vibrational state of the diatom, $f$ is the final vibrational state, and $n$ is defined as the minimum of $i$ and $f$. The parameter $\varepsilon_{\mathrm{COL}}$ is defined by Eq. 3 where $S_{\mathrm{VT}}$ is a steric factor, $\omega$ is the oscillator frequency, $\tilde{m}$ is the collision reduced mass, $\mu$ is the oscillator reduced mass, $\gamma$ is the oscillator mass ratio, $h$ is the Planck constant, $\bar{v}$ is the symmetrized relative velocity, and $\alpha$ characterizes the intermolecular potential.

$$
\begin{gathered}
P_{\mathrm{VT}}\left(i \rightarrow f, \varepsilon_{\mathrm{COL}}\right)=i ! f ! \varepsilon_{\mathrm{COL}}^{i+f} \exp \left(-\varepsilon_{\mathrm{COL}}\right)\left|\sum_{r=0}^{n} \frac{(-1)^{r}}{r !(i-r) !(f-r) !} \frac{1}{\varepsilon_{\mathrm{COL}}^{r}}\right|^{2} \\
\varepsilon_{\mathrm{COL}}=S_{\mathrm{VT}} \frac{4 \pi^{3} \omega\left(\tilde{m}^{2} / \mu\right) \gamma}{\alpha^{2} h} \sinh ^{-2}\left(\frac{\pi \omega}{\alpha \bar{v}}\right)
\end{gathered}
$$

For diatom-diatom collisions, the probability of vibrational relaxation is described by Eq. 4 using the same definitions as the atom-diatom collision and $C_{a, b}^{c}$ is the transformation matrix ${ }^{11}$ whose coefficients are defined by Eq. 5. The parameter $\rho_{\mathrm{COL}}$ is defined by Eq. 6 where $S_{\mathrm{Vv}}$ is a steric factor, and $\omega_{n}$ is the oscillator frequency of molecule $n$.

$$
\begin{gathered}
P_{\mathrm{VVT}}\left(i_{1}, i_{2} \rightarrow f_{1}, f_{2}, \varepsilon_{\mathrm{COL}}, \rho_{\mathrm{COL}}\right)= \\
\left|\sum_{r=0}^{n}(-1)^{i_{1}+i_{2}-r} C_{r+1, i_{2}+1}^{i_{1}+i_{2}} C_{r+1, f_{2}+1}^{f_{1}+f_{2}} \exp \left(-i \rho_{\mathrm{COL}}\left(f_{1}+f_{2}-r\right)\right) P_{\mathrm{VT}}^{1 / 2}\left(i_{1}+i_{2}-r \rightarrow f_{1}+f_{2}-r, 2 \varepsilon_{\mathrm{COL}}\right)\right| \\
C_{k j}^{n}=2^{-n / 2}\left[\begin{array}{c}
n \\
k-1
\end{array}\right]^{1 / 2}\left[\begin{array}{c}
n \\
j-1
\end{array}\right]^{1 / 2} \sum_{r=0}^{j-1}(-1)^{n-1-r}\left[\begin{array}{c}
n-k+1 \\
j-r-1
\end{array}\right]\left[\begin{array}{c}
k-1 \\
r
\end{array}\right] \\
\rho_{\mathrm{COL}}=\left(S_{\mathrm{Vv}} \frac{\alpha^{2} \bar{v}^{2}}{\omega_{1} \omega_{2}}\right)^{1 / 2}
\end{gathered}
$$

In the FHO model, the vibrational cross-sections and the PVI-VEDF are inherently linked. The steric factors, $S_{\mathrm{VT}}$ and $S_{\mathrm{Vv}}$, and the parameter $\alpha$ determine the rate of vibrational relaxation, while the inherent form of the transition probability determines the PVI-VEDF. Because of the complexity of the mathematical form, Boyd and Josyula ${ }^{1}$ suggested accumulating the transition probabilities into two tables. For example, for an atom-diatom collision given an initial state, $\left(i, \varepsilon_{\mathrm{COL}}\right)$, the probability of any change in vibrational quantum, $P_{\mathrm{VT-тоL}}$, can be accumulated into the first table by summing over all possible final states as seen in Eq. 7.

$$
P_{\mathrm{VT}-\mathrm{TOL}}=\sum_{f \neq i}^{f_{\text {max }}} P_{\mathrm{VT}}\left(i \rightarrow f, \varepsilon_{\mathrm{COL}}\right)
$$
8 .

Then, a second table gives the PVI-VEDF, $f_{\mathrm{PVI}-\mathrm{VEDF}}\left(i \rightarrow f, \varepsilon_{\mathrm{COL}}\right)$, for each initial state as seen in Eq.

$$
f_{\mathrm{FHO}}=\frac{P_{\mathrm{VT}}\left(i \rightarrow f, \varepsilon_{\mathrm{COL}}, i \neq f\right)}{P_{\mathrm{VT}-\mathrm{TOL}}\left(i \rightarrow f, \varepsilon_{\mathrm{COL}}\right)}
$$

This lends itself to a similar computational algorithm used in the low-fidelity vibrational relaxation models where the first table provides a vibrational collision number that is dependent on initial conditions. If selected for a vibrationally inelastic collision, the second table provides the scaled PVI-VEDF that is sampled.

\section{Assessment of Models}

In this section, we compare the vibrational relaxation models to the quasi-classical trajectory (QCT) calculations of Braunstein and Duff ${ }^{6}$ for the $\mathrm{CO}\left(v_{i}, T_{\mathrm{ROT}}=300 \mathrm{~K}\right)+\mathrm{O} \rightarrow \mathrm{CO}\left(v_{f}, j_{f}\right)+\mathrm{O}$ system over a wide range of relative collision velocities. Both models have internal parameters that can be set to match consistent vibrational collision numbers. However, the vibrational relaxation model form dictates the PVIVEDF and therefore the evolution of the detailed vibrational relaxation process. We isolate the effects of

American Institute of Aeronautics and Astronautics

Distribution A: Approved for public release; distribution unlimited. 
the model form for a zero-dimensional vibrational relaxation case and compare the evolution of the VEDF and radiative emission predicted by each model. The section closes with a study of how the differences in the VEDF affect the predicted radiative emission spectrum.

\section{A. Initial Model Comparison}

Figures 4 and 5 compare the PVI-VEDF predicted by the FHO and LB models with the QCT data at an initial rotational temperature of $300 \mathrm{~K}$; initial vibrational levels of 0,2 , and 4 ; and relative collision velocities of $4,6,8$, and $10 \mathrm{~km} / \mathrm{s}$. In general, the LB model predicts that the probability for the final vibrational level is inversely proportional to the final vibrational level. In contrast, the FHO model generally predicts that the probability for each final state is inversely proportional with the vibrational quanta jump. In general, the LB model is in better agreement with the QCT data at lower collision energies, while the FHO model is in better agreement with the QCT data at higher collision velocities.

Figure 4(a) shows the PVI-VEDF from an initial ground vibrational state and collision velocity of $4 \mathrm{~km} / \mathrm{s}$. Here, both models and QCT results predict that almost all molecules are excited to the first two excited states, with a single quantum excitation having a probability greater than $85 \%$. However, compared to the QCT results, both vibrational relaxation models under-predict the probability of a double quanta excitation and consequently over-predict a single quanta excitation. Figures $4(\mathrm{~b})$ and $5(\mathrm{a})$ and (b) show similar trends with an increase in the probability of multiple quantum jumps that increases with the increase in relative collision velocity. The FHO and LB models tend to predict fewer multiple state transition than the QCT data, with the LB model maintaining a better level of agreement with the QCT data.

Figure 4(c) shows the PVI-VEDF for a CO molecule initially in the second excited vibrational state and collision velocity of $4 \mathrm{~km} / \mathrm{s}$. Both the LB model and QCT data predict a probability that is proportional to the final state, while the FHO model predicts a probability that is proportional to the quanta jump. Figures 4(d) and 5(c) and (d) show similar trends for both LB and FHO models with an increase in possible final vibrational states with an increase in relative collision velocity. Interestingly at the higher velocities of 8 and $10 \mathrm{~km} / \mathrm{s}$, the QCT data shows a change in trend where the probability of a post-collision state in the ground vibrational state is less than the first and third excited state. This results in the FHO model qualitatively showing the same trend as the QCT data. With the increase in available post-collision states, the FHO model begins to show local maxima that are away from the single quanta jump. Similar local maxima have been calculated with the FHO model near resonant collision velocities. ${ }^{12}$

Figures $4(\mathrm{e})$ and (f) show the PVI-VEDF from $v_{i}=4$ and collision velocity of 4 and $6 \mathrm{~km} / \mathrm{s}$, respectively. The QCT data, LB, and FHO models all predict similar trends as they do for the collision process from $v_{i}=2$. Figures $5(\mathrm{e})$ and (f) show the PVI-VEDF from the initial fourth excited vibrational state with collision velocities of 8 and $10 \mathrm{~km} / \mathrm{s}$, respectively. Again, we see very similar trends from the second excited vibrational state. Now, the FHO model predicts more pronounced local maxima away from the single quanta jump. We also see that these maxima can shift to higher states as the relative collision velocity increases. For quenching processes, the QCT data predicts slight maxima, however the QCT data always monotonically

decrease as the vibrational energy level jump increases for all excitation processes. As with all other collision classes studied, the LB model consistently predicts that the probability is proportional to the final vibrational state.

\section{B. Vibrational Relaxation Comparison}

The effects of the model form can be isolated from the collision number using numerical simulations. Both the LB and FHO models are used within the DSMC method to predict adiabatic relaxation. A zero dimensional, unsteady configuration in which the energy modes of the gas are initialized to Boltzmann distributions specified by a mode temperature is used to model the basic vibrational relaxation process. The initial conditions for the adiabatic relaxation case presented here include an initial pressure of $p=101,325 \mathrm{~Pa}$, translational temperature of $T_{\mathrm{TRA}}=5,000 \mathrm{~K}$ and vibrational temperature of $T_{\mathrm{VIB}}=1,000 \mathrm{~K}$. The gas species concentration consists of $50 \% \mathrm{CO}$ and $50 \% \mathrm{O}$ by mole fraction. Vibrational relaxation between $\mathrm{CO}$ and $\mathrm{CO}$ is suppressed to isolate the effects of the $\mathrm{CO}+\mathrm{O}$ collisions. In addition, the effects of radiative decay on the VEDF is ignored. Both simulations include consistent rotational relaxation models for the $\mathrm{CO}+\mathrm{O}$ and $\mathrm{CO}+\mathrm{CO}$ collision classes.

Figures 6 and 8 show the time evolution of the translational and vibrational temperature obtained with DSMC using both the FHO and LB models. Here, the translational temperature is calculated using thermal 


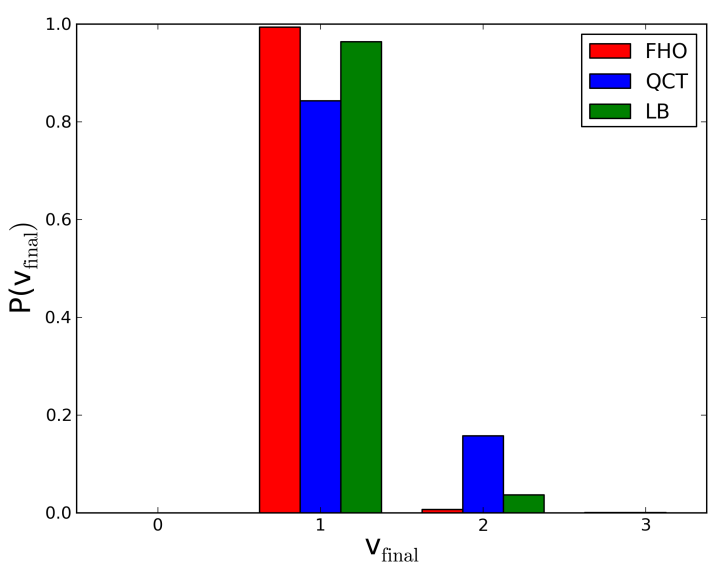

(a) $v_{\mathrm{i}}=0, g_{\text {rel }}=4 \mathrm{~km} / \mathrm{s}$

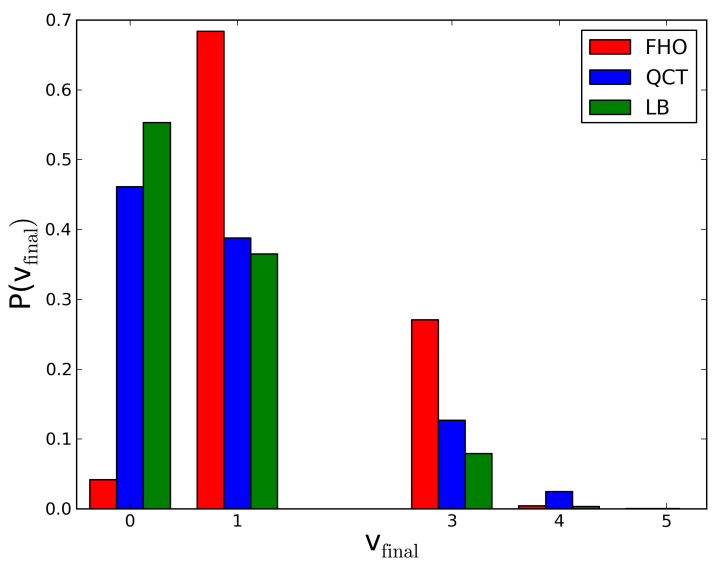

(c) $v_{\mathrm{i}}=2, g_{\text {rel }}=4 \mathrm{~km} / \mathrm{s}$

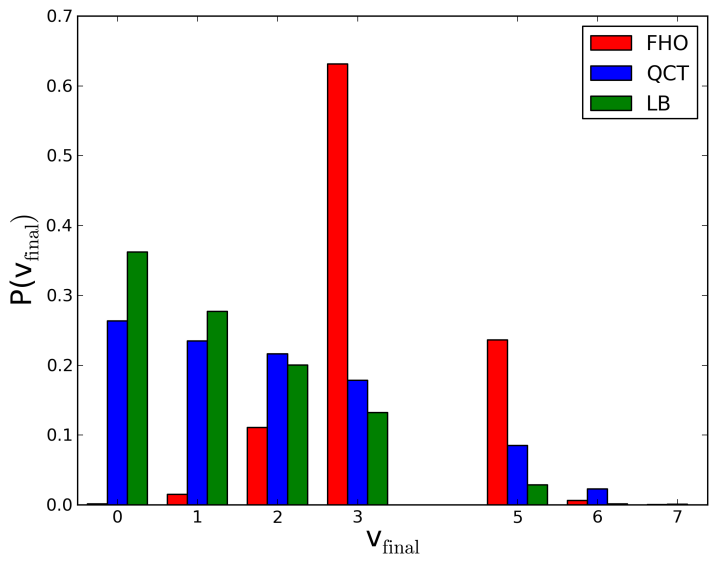

(e) $v_{\mathrm{i}}=4, g_{\text {rel }}=4 \mathrm{~km} / \mathrm{s}$

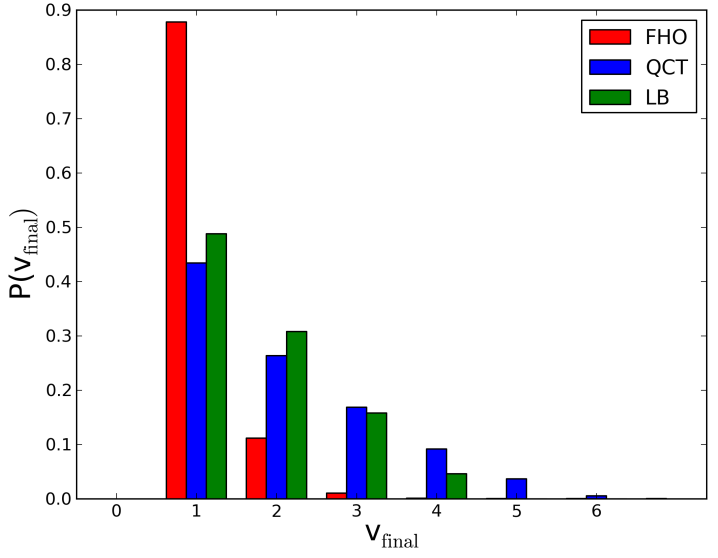

(b) $v_{\mathrm{i}}=0, g_{\text {rel }}=6 \mathrm{~km} / \mathrm{s}$

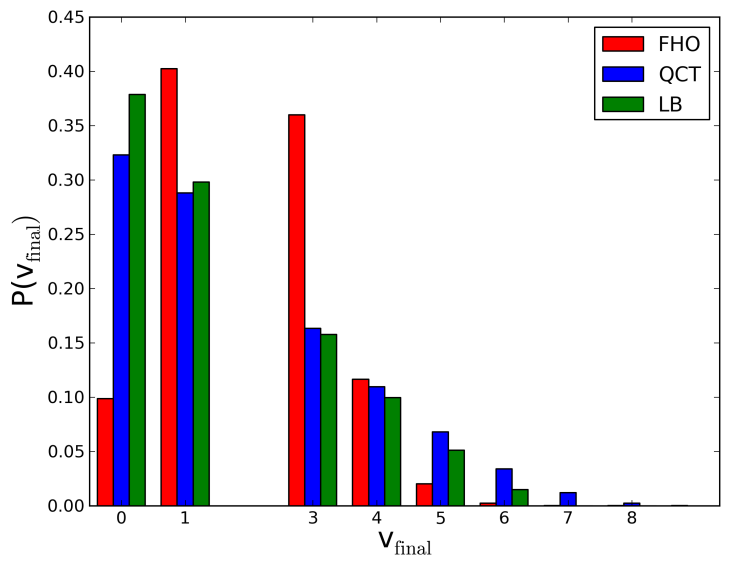

(d) $v_{\mathrm{i}}=2, g_{\text {rel }}=6 \mathrm{~km} / \mathrm{s}$

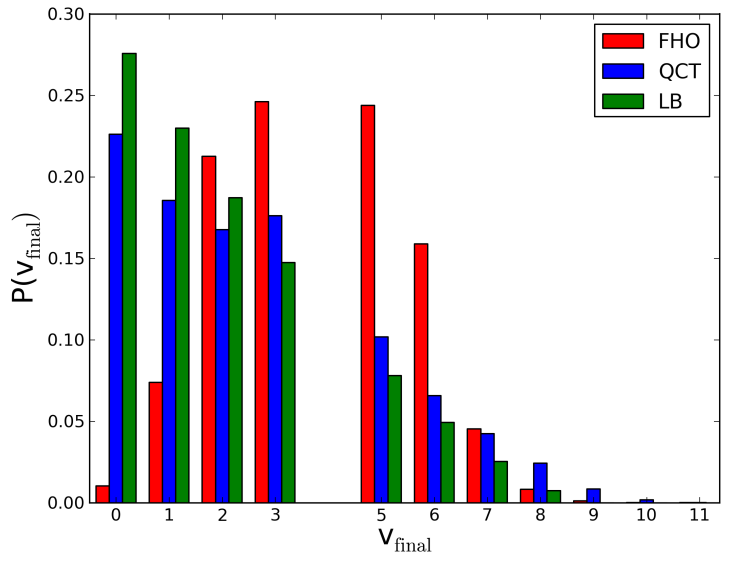

(f) $v_{\mathrm{i}}=4, g_{r e l}=6 \mathrm{~km} / \mathrm{s}$

Figure 4: Predicted PVI-VEDF from vibrational relaxation models and QCT results with an initial rotational temperature of $300 \mathrm{~K}$; initial vibrational level of 0 (top), 2 (middle) and 4 (bottom); and relative velocity of $4 \mathrm{~km} / \mathrm{s}$ (left) and $6 \mathrm{~km} / \mathrm{s}$ (right).

\section{8 of 15}

American Institute of Aeronautics and Astronautics

Distribution A: Approved for public release; distribution unlimited. 


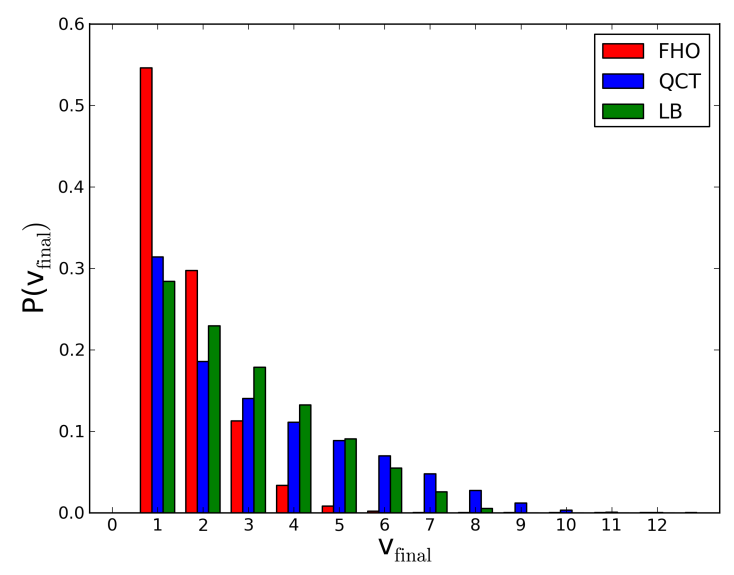

(a) $v_{\mathrm{i}}=0, g_{\text {rel }}=8 \mathrm{~km} / \mathrm{s}$

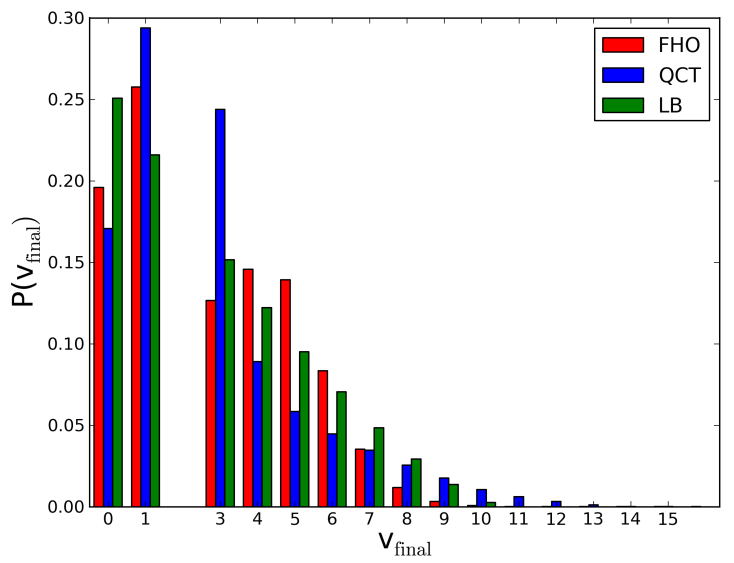

(c) $v_{\mathrm{i}}=2, g_{r e l}=8 \mathrm{~km} / \mathrm{s}$

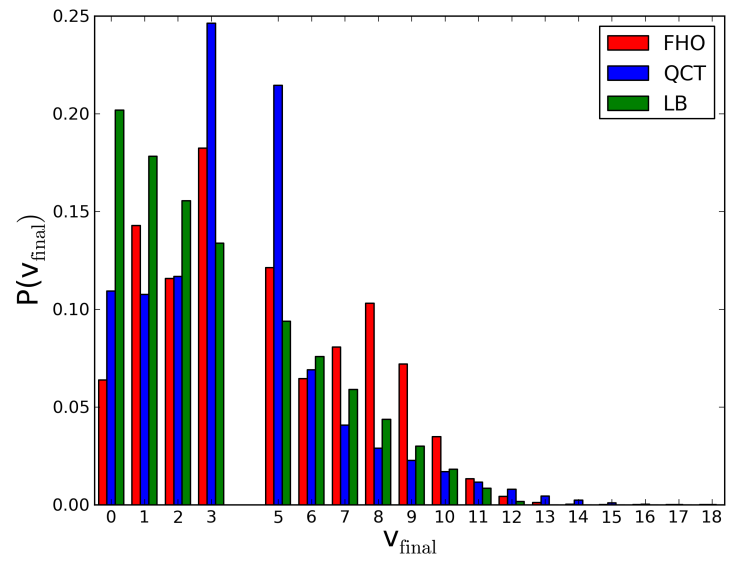

(e) $v_{\mathrm{i}}=4, g_{\text {rel }}=8 \mathrm{~km} / \mathrm{s}$

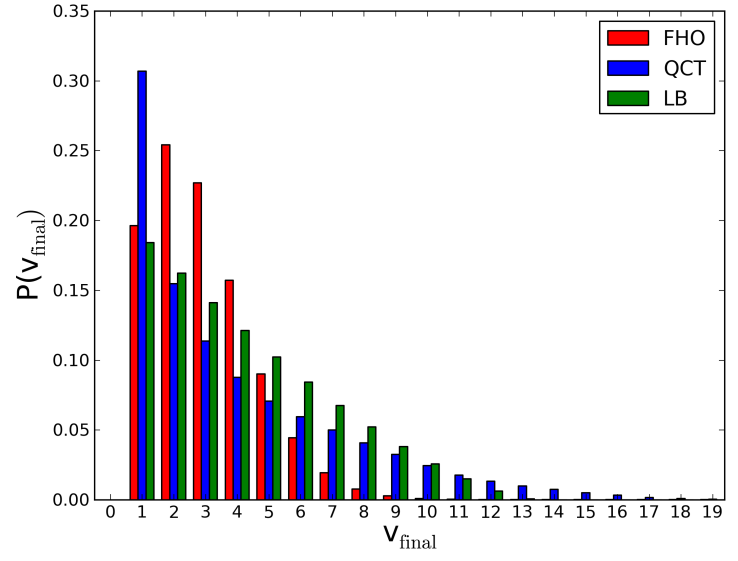

(b) $v_{\mathrm{i}}=0, g_{r e l}=10 \mathrm{~km} / \mathrm{s}$

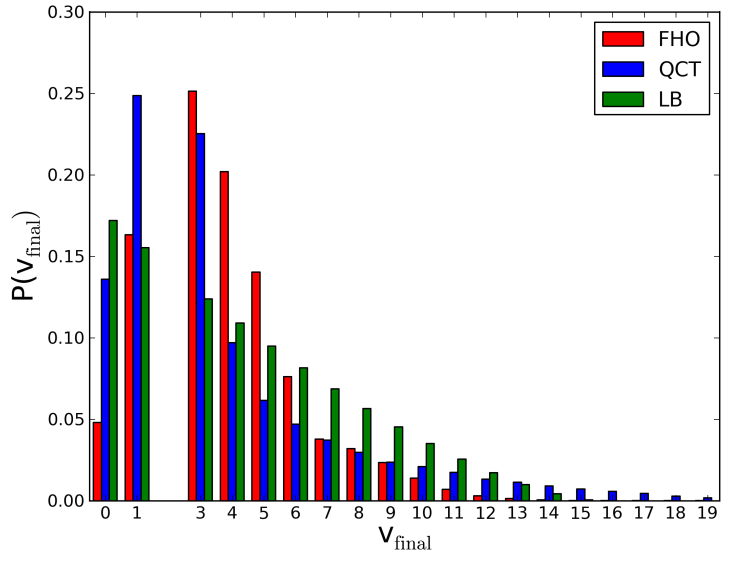

(d) $v_{\mathrm{i}}=2, g_{r e l}=10 \mathrm{~km} / \mathrm{s}$

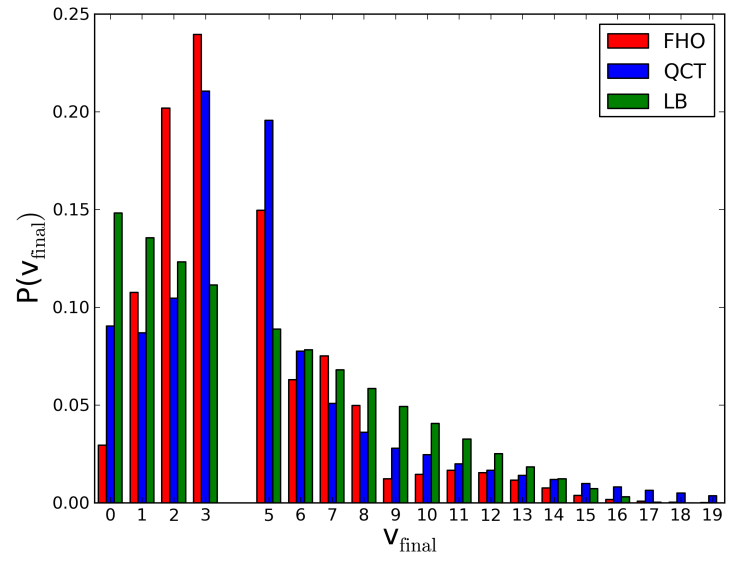

(f) $v_{\mathrm{i}}=4, g_{r e l}=10 \mathrm{~km} / \mathrm{s}$

Figure 5: Predicted PVI-VEDF from vibrational relaxation models and QCT results with an initial rotational temperature of $300 \mathrm{~K}$; initial vibrational level of 0 (top), 2 (middle) and 4 (bottom); and relative velocity of $8 \mathrm{~km} / \mathrm{s}$ (left) and $10 \mathrm{~km} / \mathrm{s}$ (right). 
velocity components as seen in Eq. 9 where $c$ is the particle velocity, $m$ is the particle mass, and $k$ is the Boltzmann constant. The vibrational temperature is calculated using the average vibrational energy per molecule, $\bar{e}_{v i b}$, as shown in Eq. 10 where $g_{i}$ is the degeneracy, and $e_{v i b, i}$ is the vibrational energy of level $i$. For this study, the collision numbers for the LB model are selected to match the macroscopic relaxation predicted by the FHO model. Indeed, Figure 6 shows that careful collision number selection can match the macroscopic temperatures predicted using each model over the entire relaxation process. It is important to note, that although the time-scales in the selected problem are too short for available measurement techniques, these time-scales increase through a decrease of the initial pressure, and therefore, increase in the collision time-scale.

$$
\begin{gathered}
T_{\mathrm{TRA}}=\frac{m}{3 k}\left[\bar{c}^{2}-\bar{c}^{2}\right] \\
\bar{e}_{v i b}=\frac{\sum_{i=1}^{n_{\text {levels }}} g_{i} e_{v i b, i} \exp \left(-e_{v i b, i} / k T_{v i b}\right)}{\sum_{i=1}^{n_{\text {levels }}} g_{i} \exp \left(-e_{v i b, i} / k T_{v i b}\right)}
\end{gathered}
$$

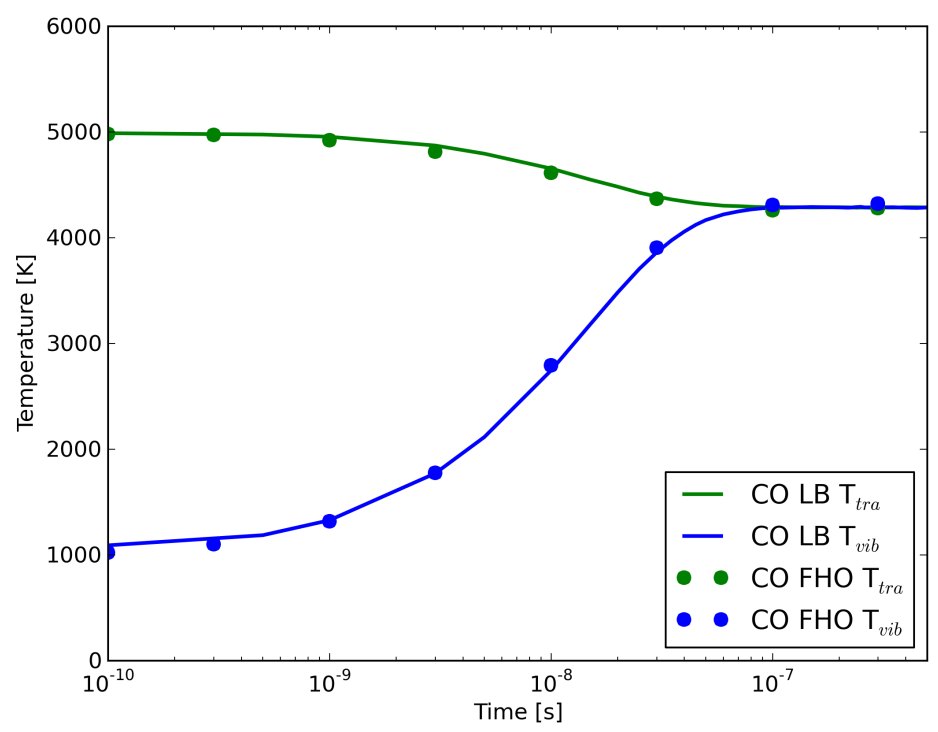

Figure 6: Evolution of adiabatic vibrational relaxation (excitation) using LB and FHO vibrational models within the DSMC method.

Despite the excellent agreement in the macroscopic temperatures, the form dictated by the FHO and LB models result in a pronounced difference in the prediction of the vibrational energy distribution function (VEDF). For example, Figures 7 and 8 (left) show the evolution of the VEDF using each model. For reference, the equilibrium Boltzmann VEDF is shown at the bulk vibrational temperature. As the VEDF transitions from the low to high temperature, both the FHO and LB models predict a bi-modal distribution with a large percentage of low energy particles and a high energy tail. Compared to the FHO model, the LB model predicts a larger change in slope of the VEDF that can described as the mode temperature. This is due to a larger disparity in the mode temperatures using the LB model and results in the FHO model predicting higher populations of the ground, first, and second excited states, while the LB model predicts higher populations in the third and higher excited states. Both models reach an equilibrium VEDF that can be described by a single temperature. This apparent bi-modal VEDF can be characterized by fitting each VEDF to the functional form shown in Eq. 11 where two temperatures, $T_{v i b, 1}$ and $T_{v i b, 2}$, and relative populations, $n_{1}$ and $n_{2}$, are selected to fit each sampled distribution function, $f_{\mathrm{VIB}-\mathrm{SAmple}}$. These fits are also shown in Figures 7 and 8(left).

$$
f_{\mathrm{VIB}-\mathrm{SAMPLE}} \approx f_{\mathrm{VIB}-\mathrm{FIT}} \sim n_{1} e^{\frac{-E_{v i b}}{k T_{v i b, 1}}}+n_{2} e^{\frac{-E_{v i b}}{k T} v i b, 1}
$$


Tables 1 and 2 list the coefficients for the VEDF fits at each time-sample slice for the LB and FHO models, respectively. The LB model predicts a VEDF that consists of two populations that are at the initial and final temperatures with a steady depletion of one to the other. Even at short times, the high temperature tail can be described with a temperature that is within $10 \%$ of the final temperature. In contrast, the FHO model predicts a VEDF that is more gradual in the transition of modes with both mode temperatures increasing as the bulk vibrational temperature approaches equilibrium.

The distribution of vibrational energy is used to predict the spectral emission. The fit parameters are used to construct a gas mixture for a line of sight that runs through a uniform gas. These lines of sight are provided to an approximate radiative transfer algorithm to predict IR emission for fundamental and hot band emission at moderate spectral resolution. ${ }^{13}$ This work assumes that the gas is optically thin. The evolution of the spectral emission for the fundamental and first overtone can be seen in Figures 7 and 8(right). Even at the initial sample slice of $1 \times 10^{-10} \mathrm{~s}$, the predicted emissive spectrum from the FHO and LB models differ. The differences can be traced back to the VEDF which is shown to the left of each spectrum. For example, the FHO VEDF results in a higher spectrum across most of the fundamental transition states $(\sim 1,500-2,300$ $\mathrm{cm}^{-1}$ ) where the $1 \rightarrow 0$ emission dominates the spectra. In this region, the peaks for all upper vibrational transitions, e.g. $2 \rightarrow 1 ; 3 \rightarrow 2$; etc., are combined with the P-branch of the $1 \rightarrow 0$ transition. The first overtone $\left(\sim 3,000-4,400 \mathrm{~cm}^{-1}\right)$ clearly shows the bandhead structure. This structure shows clear emission peaks for the $2 \rightarrow 0\left(\sim 4,370 \mathrm{~cm}^{-1}\right), 3 \rightarrow 1\left(\sim 4,315 \mathrm{~cm}^{-1}\right), 4 \rightarrow 2\left(\sim 4,260 \mathrm{~cm}^{-1}\right)$, etc transitions. As the LB VEDF predicts higher populations for most excited vibrational states, it predicts higher radiance than the FHO model for most of the overtone emission, outside of the $2 \rightarrow 0$ peak. This is expected since the FHO VEDF predicts a higher population for the second excited vibrational state.

These trends continue throughout the relaxation process. Until both VEDFs reach the Boltzmann distribution function at equilibrium, the FHO always predicts a higher population in the first excited vibrational state, which results in the FHO predicting a higher radiance over most of the fundamental band. The LB VEDF predicts higher populations for all most excited states which results in a higher emission over most of the overtone band. Although both models differ from the Boltzmann VEDF at the bulk vibrational temperature, the LB model predicts a higher level of bi-modal behavior throughout the relaxation process. At equilibrium, both models predict the same Boltzmann VEDF which results in a very high level of agreement in the predicted spectra.

Table 1: Bi-Modal VEDF LB Fit Coefficients

\begin{tabular}{|c|c|c|c|c|}
\hline Time $[\mathrm{s}]$ & $n_{1} / n_{\mathrm{TOT}}$ & $T_{\mathrm{VIB}, 1}[\mathrm{~K}]$ & $n_{2} / n_{\text {Tот }}$ & $T_{\mathrm{VIB}, 2}[\mathrm{~K}]$ \\
\hline \hline $1 \times 10^{-10}$ & .96 & 1009 & .04 & 3981 \\
\hline $1 \times 10^{-9}$ & .88 & 1029 & .12 & 4001 \\
\hline $3 \times 10^{-9}$ & .65 & 1117 & .35 & 4100 \\
\hline $1 \times 10^{-8}$ & .22 & 1249 & .78 & 4212 \\
\hline $3 \times 10^{-8}$ & 1 & 4288 & & \\
\hline $3 \times 10^{-7}$ & 1 & 4300 & & \\
\hline
\end{tabular}

Table 2: Bi-Modal VEDF FHO Fit Coefficients

\begin{tabular}{|c|c|c|c|c|}
\hline Time $[\mathrm{s}]$ & $n_{1} / n_{\mathrm{TOT}}$ & $T_{\mathrm{VIB}, 1}[\mathrm{~K}]$ & $n_{2} / n_{\mathrm{TOT}}$ & $T_{\mathrm{VIB}, 2}[\mathrm{~K}]$ \\
\hline \hline $1 \times 10^{-10}$ & .77 & 1018 & .23 & 2064 \\
\hline $1 \times 10^{-9}$ & .60 & 1201 & .40 & 2476 \\
\hline $3 \times 10^{-9}$ & .19 & 1418 & .81 & 3089 \\
\hline $1 \times 10^{-8}$ & .05 & 1818 & .95 & 4010 \\
\hline $3 \times 10^{-8}$ & .01 & 155 & .99 & 4362 \\
\hline $3 \times 10^{-7}$ & .02 & 145 & .98 & 4396 \\
\hline
\end{tabular}

11 of 15

American Institute of Aeronautics and Astronautics

Distribution A: Approved for public release; distribution unlimited. 


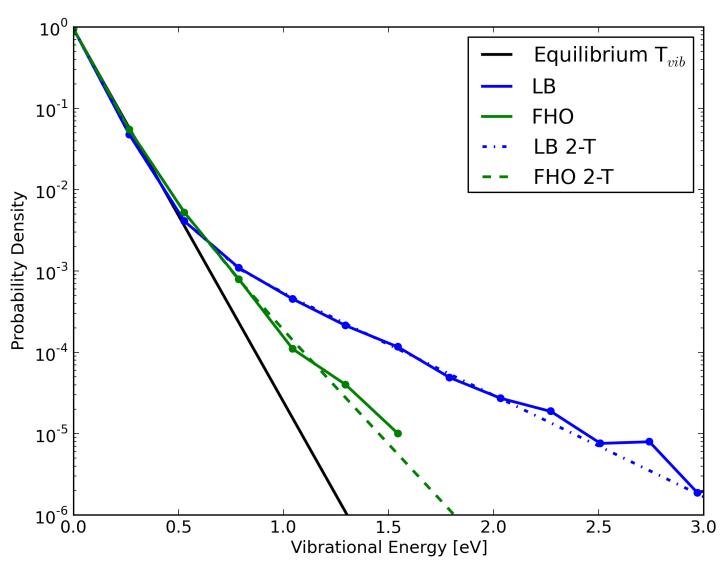

(a) VEDF, $t=1 \times 10^{-10} \mathrm{~s}$

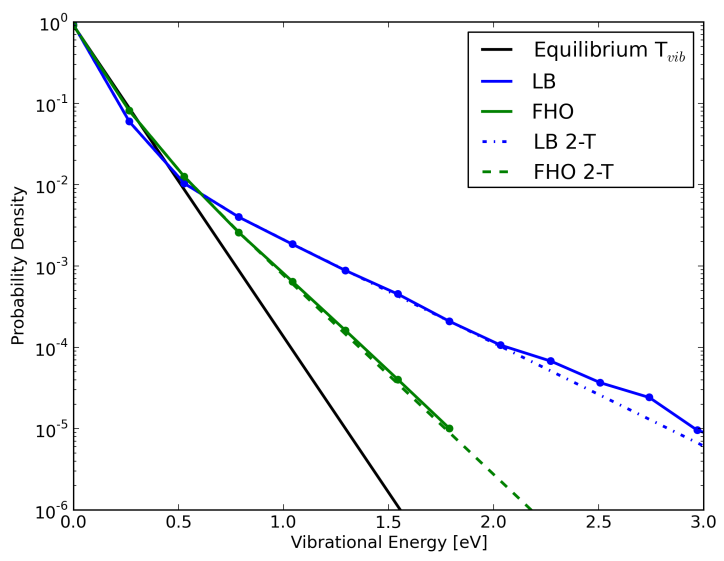

(c) VEDF, $t=1 \times 10^{-9} \mathrm{~s}$

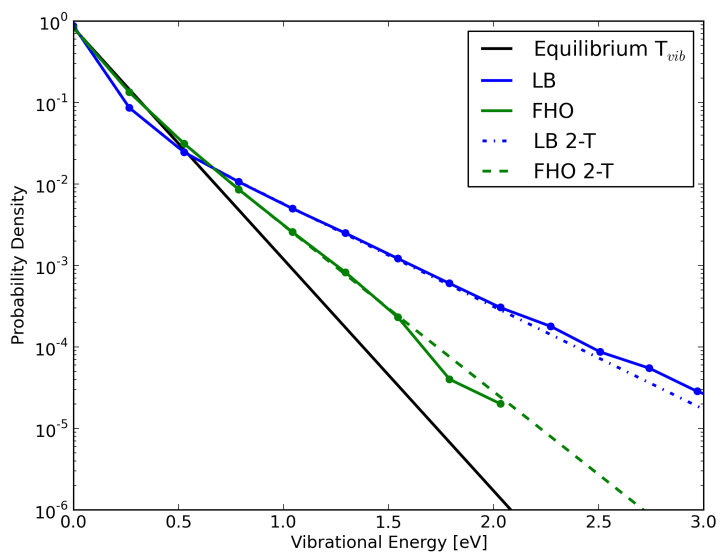

(e) VEDF, $t=3 \times 10^{-9} \mathrm{~s}$

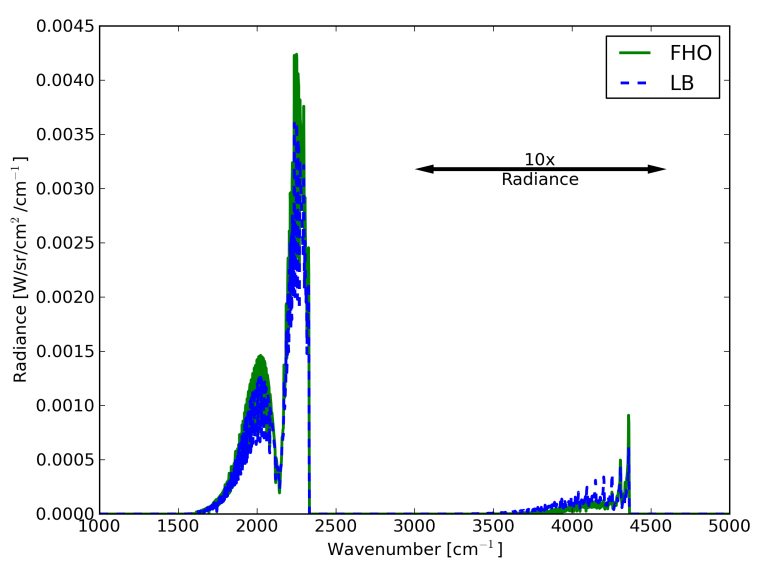

(b) Spectra, $t=1 \times 10^{-10} \mathrm{~s}$

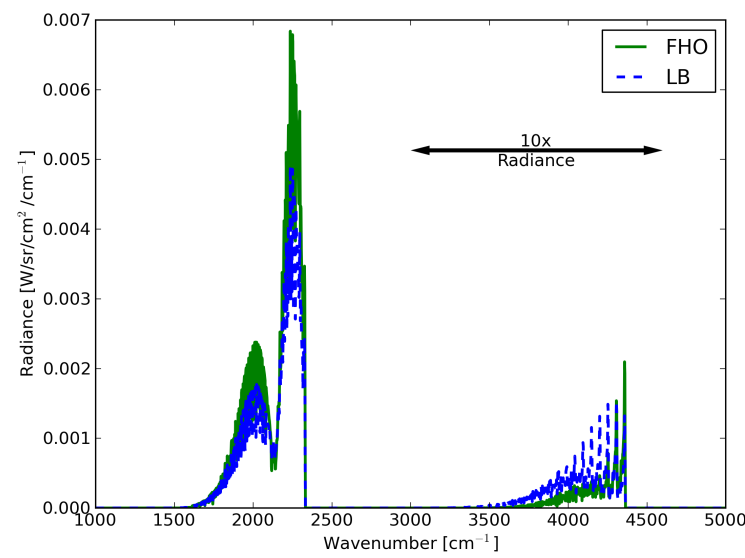

(d) Spectra, $t=1 \times 10^{-9} \mathrm{~s}$

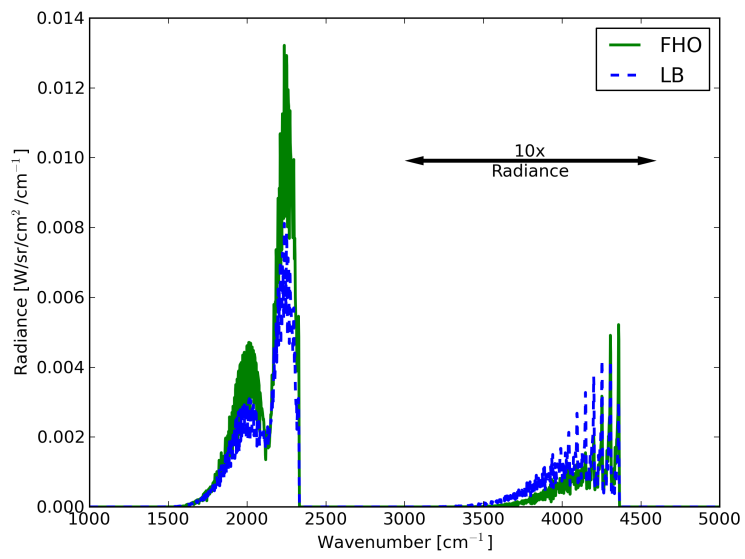

(f) Spectra, $t=3 \times 10^{-9} \mathrm{~s}$

Figure 7: Evolution of the VEDF (left) and radiative emission (right) using FHO and LB vibrational models for adiabatic relaxation.

American Institute of Aeronautics and Astronautics

Distribution A: Approved for public release; distribution unlimited. 


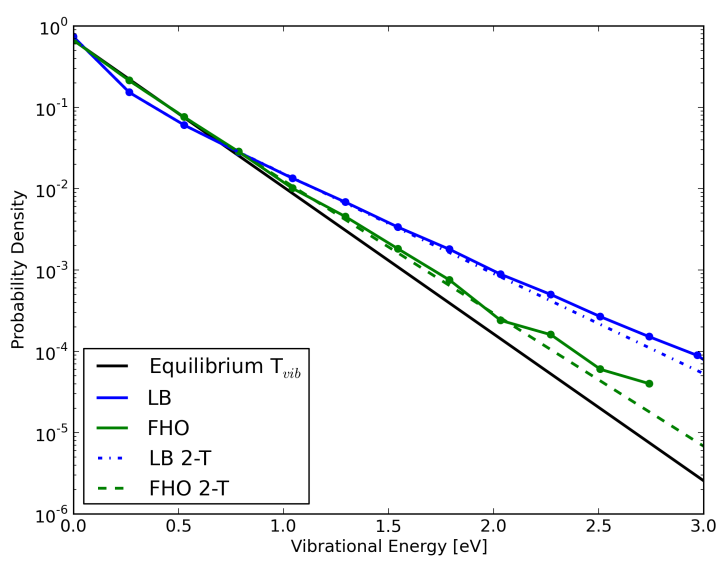

(a) VEDF, $t=1 \times 10^{-8} \mathrm{~s}$

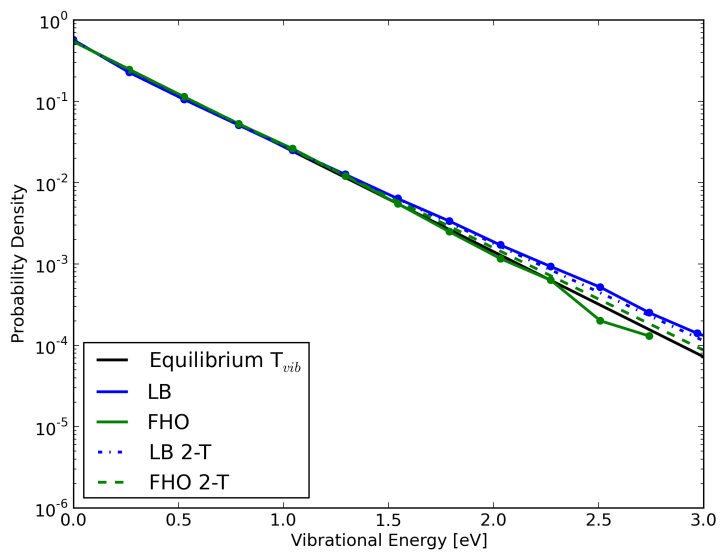

(c) VEDF, $t=3 \times 10^{-8} \mathrm{~s}$

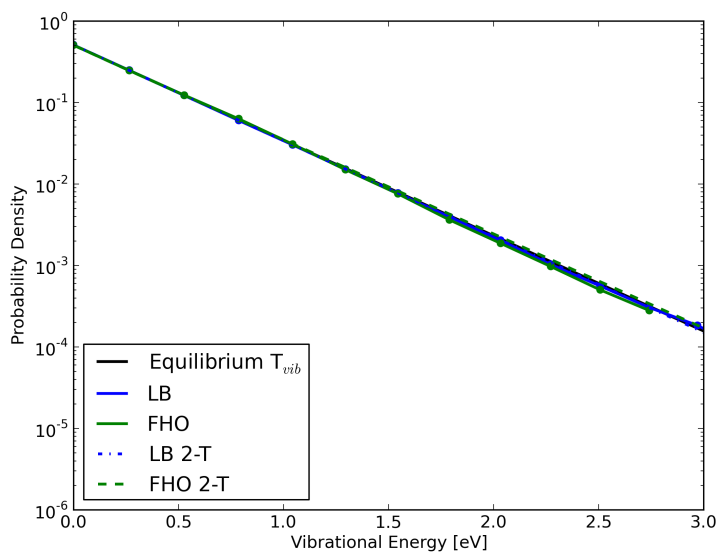

(e) VEDF, $t=3 \times 10^{-7} \mathrm{~s}$

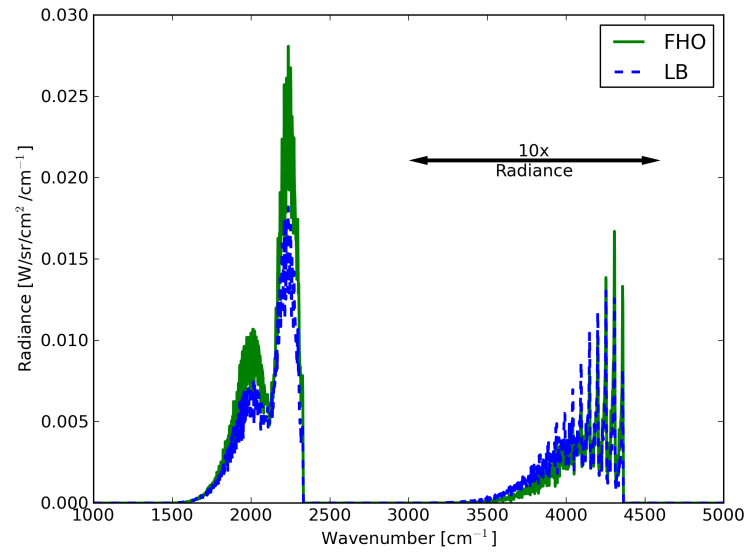

(b) Spectra, $t=1 \times 10^{-8} \mathrm{~s}$

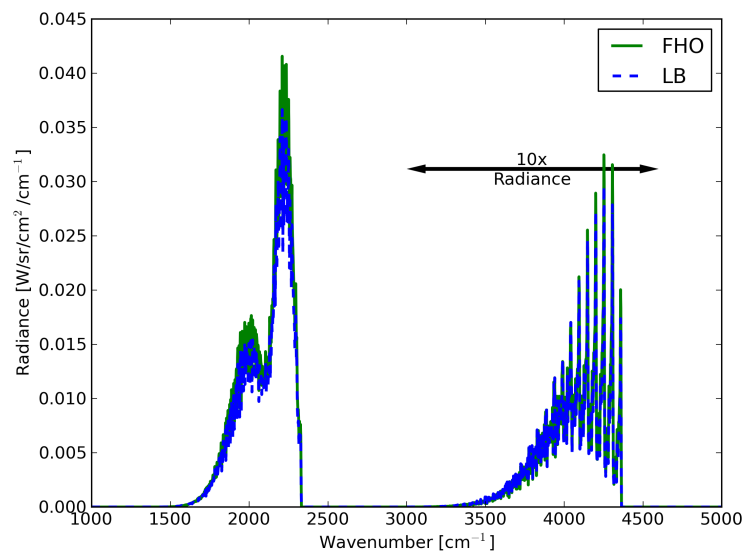

(d) Spectra, $t=3 \times 10^{-8} \mathrm{~s}$

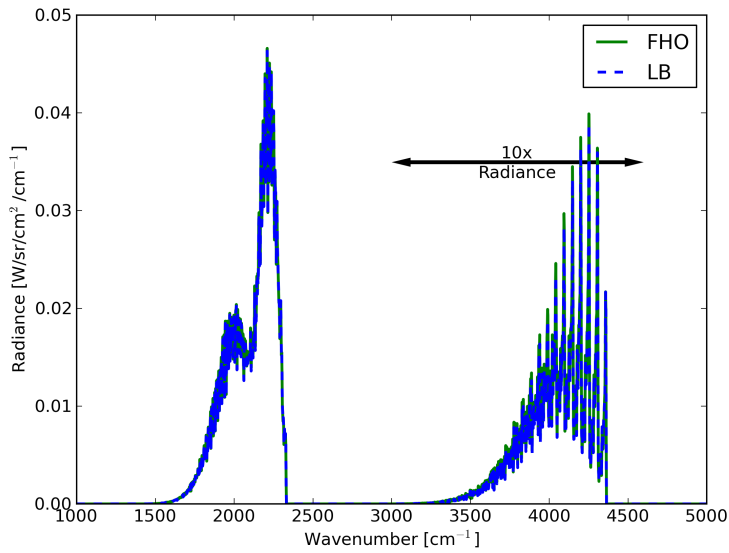

(f) Spectra, $t=3 \times 10^{-7} \mathrm{~s}$

Figure 8: Evolution of the VEDF (left) and radiative emission (right) using FHO and LB vibrational models for adiabatic relaxation (continued). 


\section{Summary and Conclusions}

The form of two vibrational relaxation models that are commonly used in DSMC and CFD simulations have been compared. In particular, this work focused on examining the effect of the model form for both the Larsen-Borgnakke (LB) and Force Harmonic Oscillator (FHO) vibrational relaxation models and the effect of model form on the predicted vibrational energy distribution function (VEDF) and radiative emission spectra. Available ro-vibrationally resolved cross sections from state of the art QCT calculations were presented for the $\mathrm{CO}\left(v_{i}, T_{\mathrm{ROT}}=300 \mathrm{~K}\right)+\mathrm{O} \rightarrow \mathrm{CO}\left(v_{f}, j_{f}\right)+\mathrm{O}$ system. This data was reduced to post-vibrationally inelastic VEDF (PVI-VEDF) for direct comparison with each model predictions over a wide range of collision energies. Comparison between each model with the QCT data showed that the LB model is in better agreement with the QCT data for lower collision velocities, while the FHO model is in better agreement for high collision velocities. Each model was used within the DSMC method for an adiabatic thermal relaxation simulation. Even when the collision numbers were selected to predict the same bulk temperature relaxation profile, the LB and FHO models predicted very different VEDFs. Both models predicted bi-modal VEDFs, however the evolution of the bi-modal temperatures and populations were different due to the model form. The disparity in the predicted VEDF resulted in differences in the predicted radiative emission spectra.

Future work will aim to extend the evaluation of each model with additional available high fidelity chemistry data and study the effect of each model on a wide range of compression and expansion flows. By leveraging recent developments that use classical trajectory calculations directly within the DSMC collision kernel, ${ }^{14}$ the vibrational relaxation models can be compared to QCT predictions for problems similar to the test case used in this paper. Alternatively, the QCT results may be integrated into the DSMC method with the LB model. Reference 15 provides an example of past success with this approach. In addition, study of the effect of the model form on chemistry would provide further understanding of coupled vibrationdissociation processes that are important in many high altitude flows. Finally, further development of vibrational relaxation models depends on high quality state-resolved experimental measured data. These initial results have shown that each model results in significant differences in a measurable optical quantity. We note that, although the time-scales for the relaxation process in this study are quite small, they can be increased through a reduction in pressure and the subsequent increase of the collision time-scale. Spectrallyresolved measurements for similar cases under non-equilibrium conditions, such as in shock tube experiments can provide further insight into inelastic collision processes.

\section{Acknowledgments}

The authors gratefully acknowledge Tom Smith (AFRL/RQRS) for continued direction and guidance. Funding for this work was provided by contract \#HQ0147-12-C-7140.

\section{References}

${ }^{1}$ Boyd, I. D. and Josyula, E., "State resolved vibrational relaxation modeling for strongly nonequilibrium flows," Physics of Fluids, Vol. 23, No. 5, 2011, pp. 057101.

${ }^{2}$ Larsen, P. S. and Borgnakke, C., "Statistical Collision Model for Simulating Polyatomic Gas with Restricted Energy Exchange," Soviet Powder Metallurgy and Metal Ceramics (English translation of Poroshkovaya Metallurgiya), 1974, pp. 9-45.

${ }^{3}$ Bird, G. A., Molecular Gas Dynamics and the Direct Simulation of Gas Flows, Clarendon Press, 1994.

${ }^{4}$ Adamovich, I. V., Macheret, S. O., Rich, J. W., and Treanor, C. E., "Vibrational Energy Transfer Rates Using a Forced Harmonic Oscillator Model," Journal of Thermophysics and Heat Transfer, Vol. 12, No. 1, Jan. 1998, pp. 57-65.

${ }^{5}$ Billing, G. D. and Fisher, E. R., "VV and VT Rate Coefficients in N2 by a Quantum-Classical Model," Chemical Physics, Vol. 43, No. 395-401, 1979, pp. 395-401.

${ }^{6}$ Braunstein, M. and Duff, J. W., "Electronic structure and dynamics of O+CO collisions," Journal of Chemical Physics, Vol. 112, No. 6, 2000, pp. 2736-2745.

${ }^{7}$ Brunsvold, A. L., Upadhyaya, H. P., Zhang, J., Cooper, R., Minton, T. K., Braunstein, M., and Duff, J. W., "Dynamics of Hyperthermal Collisions of O(3P) with CO," Journal of Physical Chemistry, Vol. 112, 2008.

${ }^{8}$ Deschenes, T. R. and Boyd, I. D., "Extension of a Modular Particle-Continuum Method to Vibrationally Excited, Hypersonic Flows," AIAA Journal, Vol. 49, No. 9, 2011, pp. 1951-1959.

${ }^{9}$ Vijayakumar, P., Sun, Q., and Boyd, I. D., "Vibrational-translational energy exchange models for the direct simulation Monte Carlo method," Physics of Fluids, Vol. 11, No. 8, Aug. 1999, pp. 2117-2126.

${ }^{10}$ Bergemann, F. and Boyd, I. D., "New Discrete Vibrational Energy Method for the Direct Simulation Monte Carlo Method," Rarefied Gas Dynamics, Progress in Astronautics and Aeronautics, 1994, pp. 174-180.

${ }^{11}$ Zelechow, A., Rapp, D., and Sharp, T. E., "Vibrational-Vibrational-Translational Energy Transfer between Two Diatomic Molecules," The Journal of Chemical Physics, Vol. 49, No. 1, 1968, pp. 286.

\section{4 of 15}

American Institute of Aeronautics and Astronautics Distribution A: Approved for public release; distribution unlimited. 
${ }^{12}$ Weaver, A. B., Ayyaswamy, V., and Alexeenko, A., Implementation Challenges and Performance of Forced Harmonic Oscillator Model in Direct Simulation Monte Carlo, American Institute of Aeronautics and Astronautics, 2013/11/15 2013.

${ }^{13}$ Sundberg, R. L., Duff, J. W., and Bernstein, L. S., "Nonequilibrium infrared emission model for the wake flow of re-entry vehicles," Journal of Spacecraft and Rockets, Vol. 30, No. 6, 1993, pp. 731-741.

${ }^{14}$ Norman, P., Valentini, P., and Schwartzentruber, T., "GPU-accelerated Classical Trajectory Calculation Direct Simulation Monte Carlo applied to shock waves," Vol. 247, 08 2013, pp. 153-167.

${ }^{15}$ Boyd, I. D., Bose, D., and Candler, G. V., "Trajectory calculations for the reaction H2 He," Chemical Physics Letters, Vol. 9, No. 4, July 1997, pp. 1162-1170.

American Institute of Aeronautics and Astronautics Distribution A: Approved for public release; distribution unlimited. 